\title{
Sustained Improvement in Hand Hygiene Adherence: Utilizing Shared Accountability and Financial Incentives
}

\author{
Thomas R. Talbot, MD, MPH; ${ }^{1,2}$ James G. Johnson, MD, MPH; ${ }^{1}$ Claudette Fergus, RN; ${ }^{3}$ John Henry Domenico, MS; ${ }^{4}$ \\ William Schaffner, MD; ${ }^{1,2}$ Titus L. Daniels, MD, MPH; ${ }^{1}$ Greg Wilson, MD; ${ }^{5}$ Jennifer Slayton, $\mathrm{RN} ;{ }^{6}$ \\ Nancye Feistritzer, RN; Gerald B. Hickson, $\mathrm{MD}^{7}$
}

овјестіvе. To evaluate the impact of an institutional hand hygiene accountability program on healthcare personnel hand hygiene adherence.

DESIGN. Time-series design with correlation analysis.

SETTING. Tertiary care academic medical center, including outpatient clinics and procedural areas.

PARTICIPANTS. Medical center healthcare personnel.

MEтноDs, A comprehensive hand hygiene initiative was implemented in 2 major phases starting in July 2009. Key facets of the initiative included extensive project planning, leadership buy-in and goal setting, financial incentives linked to performance, and use of a systemwide shared accountability model. Adherence was measured by designated hand hygiene observers. Adherence rates were compared between baseline and implementation phases, and monthly hand hygiene adherence rates were correlated with monthly rates of device-associated infection.

RESULTS. A total of 109,988 observations were completed during the study period, with a sustained increase in hand hygiene adherence throughout each implementation phase $(P<.0001)$ as well as from one phase to the next $(P<.0001)$, such that adherence greater than $85 \%$ has been achieved since January 2011 . Medical center departments were able to reclaim some rebate dollars allocated through a selfinsurance trust, but during the study period, departments did not achieve full reimbursement. Hand hygiene adherence rates were inversely correlated with device-associated standardized infection ratios $\left(R^{2}=0.70\right)$.

ConCLUSIONS. Implementation of this multifaceted, observational hand hygiene program was associated with sustained improvement in hand hygiene adherence. The principles of this program could be applied to other medical centers pursuing improved hand hygiene adherence among healthcare personnel.

Infect Control Hosp Epidemiol 2013;34(11):1129-1136

Hand hygiene $(\mathrm{HH})$ is essential in preventing healthcareassociated infections (HAIs), ${ }^{1,2}$ yet $\mathrm{HH}$ adherence among healthcare personnel (HCP) remains suboptimal. ${ }^{2,3}$ In 2009, Vanderbilt University Medical Center (VUMC) launched a system-wide initiative aimed at achieving and sustaining $\mathrm{HH}$ adherence using direct observation (including outpatient and procedural areas), an accountability structure and process, and financial incentives. This article details the VUMC HH program implementation and subsequent impact from July 2009 through August 2012.

\section{METHODS}

\section{Setting}

VUMC is a health system based in Nashville, Tennessee, that includes adult, pediatric, and psychiatric hospitals; on-

Affiliations: 1. Department of Medicine, Division of Infectious Diseases, Vanderbilt University School of Medicine, Nashville, Tennessee; 2. Department of Preventive Medicine, Vanderbilt University School of Medicine, Nashville, Tennessee; 3. Vanderbilt University Medical Center, Nashville, Tennessee;

4. Department of Biostatistics, Vanderbilt University School of Medicine, Nashville, Tennessee; 5. Department of Pediatrics, Division of Infectious Diseases, Vanderbilt University School of Medicine, Nashville, Tennessee; 6. Monroe Carell Jr. Children's Hospital at Vanderbilt, Nashville, Tennessee;

7. Vanderbilt Center for Patient and Professional Advocacy, Vanderbilt University Medical Center, Nashville, Tennessee.

Received March 7, 2013; accepted June 18, 2013; electronically published September 23, 2013.

(c) 2013 by The Society for Healthcare Epidemiology of America. All rights reserved. 0899-823X/2013/3411-0001\$15.00. DOI: 10.1086/673445 
campus outpatient clinics; and an extensive network of outpatient care sites throughout middle Tennessee. VUMC supports over 65,000 inpatient admissions, 160,000 emergency department visits, and 1.6 million ambulatory visits annually. ${ }^{4}$

\section{Baseline Program}

From 2004 to 2009, the VUMC HH program consisted of mandatory annual faculty and staff training, awareness events, and adherence monitoring via direct observation. These efforts produced limited improvement in measured $\mathrm{HH}$ adherence rates. In June 2009, VUMC leadership established the following 2 goals: (1) to improve $\mathrm{HH}$ adherence and (2) to prevent HAIs through efforts aligned with pursuit of a culture of safety. ${ }^{5}$ Because these interventions were iterative quality improvement efforts involving no protected health information, Vanderbilt institutional review board approval was not required.

\section{Launch of Expanded Program}

Implementation of the $\mathrm{HH}$ initiative was performed in phases, with planning and readiness assessment followed by the program launch and a later active accountability phase. The following section describes key elements of the first phase.

Readiness assessment and planning. Before initiating the system-wide initiative and to increase the probability of success, the $\mathrm{HH}$ team used a project bundle to direct preparation, as described previously. ${ }^{5}$ The bundle consists of 9 elements subdivided into 3 sections: learning system, people, and organizational readiness. ${ }^{5}$ The project bundle focused planners on addressing the following: defining the problem, ensuring project alignment with the organization's mission, securing financial support, defining performance and measurement objectives, and establishing leadership commitment.

Leadership goal setting. After preplanning and securing leadership commitment, improved $\mathrm{HH}$ adherence was adopted as an institutional quality improvement goal. Performance related to the goal immediately became a factor in annual performance evaluations and incentive compensation for medical center leaders.

Financial incentives via a self-insurance trust allocation rebate program. VUMC is self-insured for malpractice claims. Funding for the trust occurs through annual facility and physician allocations (premiums). Because of favorable claims experience, instead of reducing premiums, a trust rebate program was created in fiscal year (FY) 2008. The program permitted academic departments and facilities to reclaim a portion of their annual allocation by achieving leadershipendorsed safety and risk-prevention goals (with associated metrics). Rebate dollars accrued to each department or facility annually in a weighted fashion on the basis of contributions to the trust and attainment of threshold, target, or reach performance targets for up to 4 goals. In June 2009, improved $\mathrm{HH}$ adherence was included as 1 of 4 patient safety metrics in the rebate program. The $\mathrm{HH}$ component of the allocation rebate was worth up to $25 \%$ of the total rebate dollars $(2.5 \%$ of yearly premiums) distributed to departments and units on the basis of the entire medical system's performance. Modest $\mathrm{HH}$ adherence goals were set in the first year of the program (VUMC-wide adherence of $65 \%$ as a threshold goal, $75 \%$ as a target goal, and $85 \%$ as a reach goal) with the intent of increasing performance requirements each year. Rebate dollars were paid as a lump sum at the close of the fiscal year and could be used at the discretion of department leadership. Anecdotally, most dollars served to support quality and safety initiatives and provide salary support for patient safety officers. The magnitude of this financial incentive was modest. Rebates could reach as high as $2.5 \%$ of the malpractice premiums for attainment of the targeted $\mathrm{HH}$ goals. For example, for a physician whose yearly premium was $\$ 10,000.00$, the rebate amounted to $\$ 250.00$.

Expanded $\mathrm{HH}$ direct observation program. From July through October 2009, the observation program was expanded to include all inpatient and outpatient locations, including procedural areas and off-site clinics. To foster shared accountability, all location managers contributed observers to the observer pool. To limit bias, observers were required to conduct observations in a location over which they had no supervisory or employment role. Each observer was tasked with conducting an assigned number of observations on the basis of the location's clinical volume and size (20 observations per month for most areas). Each observation included $\mathrm{HH}$ adherence, the observed moment (eg, before room entry), job description of the person observed, and location. All VUMC faculty and staff were eligible for observation.

Observers attended required training on a standardized observation methodology. Although the education program emphasized the need to perform $\mathrm{HH}$ for each of the World Health Organization's 5 moments for $\mathrm{HH},{ }^{6}$ to create a sustainable and less obtrusive process for observations, we adopted a simplified measurement strategy incorporating observation of adherence before entry and upon exit of the patient environment. Initially, observers were instructed to simply observe and record $\mathrm{HH}$ adherence in an unobtrusive fashion; however, the observer role expanded as accountability interventions were added in the program's second phase (see below).

System-wide marketing campaign. In May 2010, VUMC launched a system-wide marketing campaign consisting of poster messaging and targeted talks aimed to increase $\mathrm{HH}$ awareness and its importance in preventing HAIs. A followup campaign was launched in July 2011 as a part of the program's active accountability phase.

\section{Active Accountability Phase}

Since November 2010, the program has also focused on active performance awareness and accountability based on the fol- 


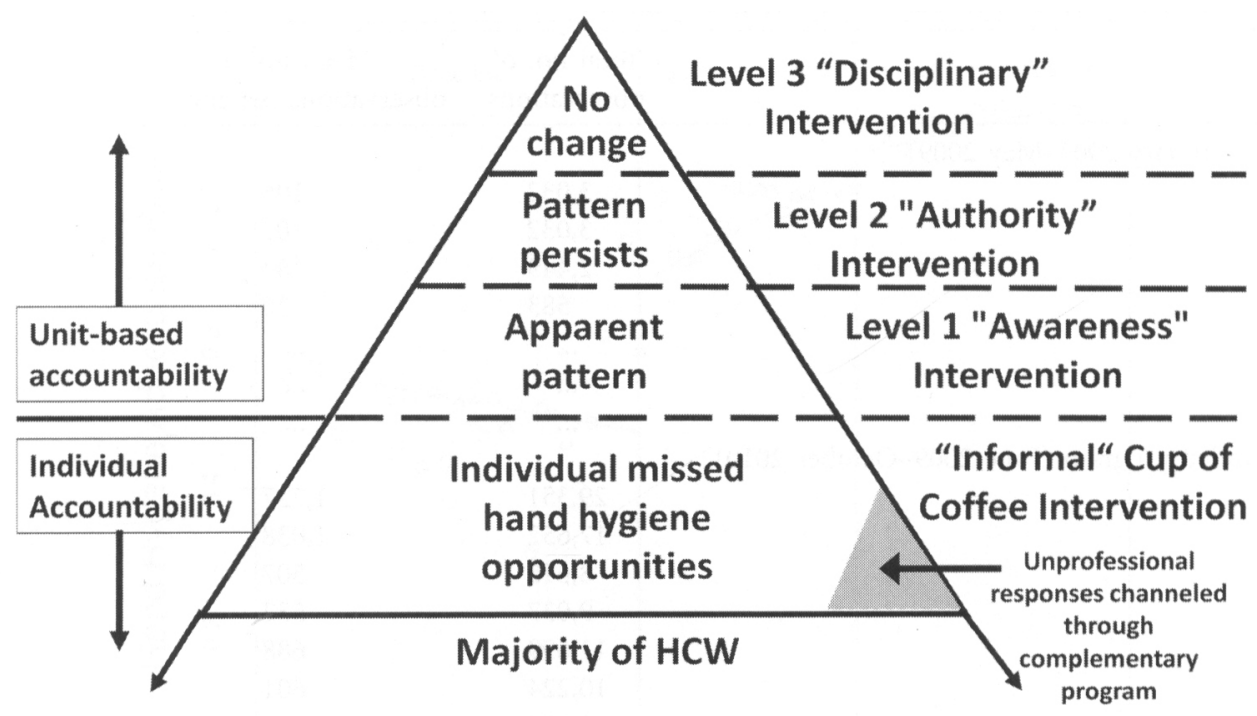

FIGURE 1. Depiction of tiered hand hygiene accountability interventions, based upon the model developed by Hickson et al. ${ }^{7} \mathrm{HCW}$, healthcare workers.

lowing steps: formation of an executive committee, locationspecific accountability interventions supported by scorecards, structured individual accountability interventions, and a continued marketing campaign. The following are descriptions of these components.

HH executive committee (HHEC). Consisting of key physician and nursing leaders, the HHEC was established to review location performance monthly and direct interventions. The HHEC meetings served to ensure $\mathrm{HH}$ remained a leadership priority and to promote transparency in accountability interventions.

Location-specific accountability interventions. At the monthly HHEC meeting, units with low adherence were identified for interventions on the basis of a system-wide $\mathrm{HH}$ intervention pyramid (Figure 1). ${ }^{7}$ The pyramid was adapted from Hickson et al, ${ }^{8,9}$ who used the associated process and method to intervene with physicians with high medical malpractice risk. As data revealed underperformance of a unit, a level 1 "awareness" intervention was initiated by the HHEC. Unit leaders (medical and nursing leader and the local quality and patient safety director) received a confidential correspondence stating that, although HHEC members knew that unit leadership shared the health system's goal of preventing HAIs, their current level of $\mathrm{HH}$ adherence was inconsistent with that goal. ${ }^{5}$ Recipients were provided a scorecard illustrating their unit's monthly and year-to-date $\mathrm{HH}$ adherence, adherence by job description (eg, physician and nurse), and a transparent comparison with other units. The letter affirmed that recipients were trusted to take actions that they thought best to address performance and that follow-up data would be provided. Following receipt of the letter, an informal meeting was held between the unit's leadership team and representatives of the
HHEC to declare confidence that the unit could reach the system's goal, to encourage dialogue regarding the unit's performance, and to address any barriers to $\mathrm{HH}$ specific to that unit. Level 2 "authority" interventions (Figure 1) were conducted when unit leaders were unable or unwilling to affect change. Differences between the level 1 and level 2 interventions were that associated department chairs or hospital directors were notified and a formal meeting among all parties was conducted to address barriers and improve performance. ${ }^{8}$ A level 3 "disciplinary" intervention (Figure 1), designed to address recalcitrant locations, required a formal corrective action plan with defined expectations, responsible parties, timeline, and consequences for failure to achieve the intended outcome. ${ }^{5}$

Structured individual accountability interventions. Initially, observers were instructed to provide no direct feedback. However, to promote culture change, direct peer-to-peer accountability was felt to be both necessary and synergistic. In November 2010, observers were trained to provide direct feedback when a $\mathrm{HH}$ opportunity was missed. Feedback occurred through what is termed a "cup of coffee" conversation (Figure 1). ${ }^{7}$ The conversation is designed as a nonjudgmental, respectful sharing of a single observation or event and is consistent with a culture of safety. The conversation could occur between any 2 parties regardless of organizational "rank." 5,7 Observers were also trained to respond to unprofessional behavior by documenting such behavior through the organization's event reporting system or using their chain of command. System leadership monitored event reporting and acted as necessary, consistent with organizational policies concerning behaviors that undermine a culture of safety. 
TA B LE 1. Hand Hygiene Adherence and Observations, Stratified by Location

\begin{tabular}{|c|c|c|c|}
\hline Variable & $\begin{array}{l}\text { Total no. of } \\
\text { observations }\end{array}$ & $\begin{array}{l}\text { Mean no. of } \\
\text { observations per month }\end{array}$ & $\begin{array}{c}\text { Mean hand } \\
\text { hygiene adherence, \% }\end{array}$ \\
\hline \multicolumn{4}{|c|}{ Baseline period (January 2007-May 2009) } \\
\hline Overall & 3,032 & 105 & 52 \\
\hline Inpatient & 3,032 & 105 & 52 \\
\hline Adult & 2,449 & 84 & 50 \\
\hline Pediatric & 583 & 20 & 62 \\
\hline Outpatient & $\ldots$ & $\ldots$ & $\ldots$ \\
\hline Adult & $\cdots$ & $\cdots$ & $\cdots$ \\
\hline Pediatric & $\ldots$ & $\ldots$ & $\ldots$ \\
\hline \multicolumn{4}{|c|}{ Launch of expanded program (June 2009-October 2010) } \\
\hline Overall & 29,351 & 1,727 & 75 \\
\hline Inpatient & 17,652 & 1,038 & 67 \\
\hline Adult & 8,615 & 507 & 68 \\
\hline Pediatric & 9,037 & 532 & 70 \\
\hline Outpatient & 11,699 & 688 & 81 \\
\hline Adult & 10,224 & 601 & 76 \\
\hline Pediatric & 1,475 & 87 & 73 \\
\hline \multicolumn{4}{|c|}{ Active accountability phase (November 2010-August 2012) } \\
\hline Overall & 80,637 & 3,665 & 89 \\
\hline Inpatient & 52,461 & 2,385 & 87 \\
\hline Adult & 21,035 & 956 & 85 \\
\hline Pediatric & 31,426 & 1,428 & 88 \\
\hline Outpatient & 28,176 & 1,281 & 93 \\
\hline Adult & 21,544 & 979 & 94 \\
\hline Pediatric & 6,632 & 301 & 93 \\
\hline
\end{tabular}

\section{Outcome Measures}

$\mathrm{HH}$ adherence was tracked using numerator and denominator data from individually recorded observations recorded through a web-based interface (REDCap Software, Nashville). Adherence was calculated as the number of adherent $\mathrm{HH}$ opportunities divided by the total opportunities observed. To examine an association between improved $\mathrm{HH}$ adherence and HAI reduction, existing institutional HAI surveillance data were used. HAI surveillance was performed prospectively by independent infection preventionists using standardized definitions for HAIs. ${ }^{10}$

\section{Statistical Analysis}

$\mathrm{HH}$ adherence data were compared using segmented regression analysis for the following phases, defined a priori: baseline period (January 2007-May 2009), program launch (June 2009-October 2010), and active accountability phase (November 2010-August 2012). F statistics were then calculated using $R$, version 2.15 .1 to evaluate differences between time periods $(P<.05$ was considered to be statistically significant). ${ }^{11,12}$ A correlation analysis between improved $\mathrm{HH}$ adherence and HAI infection reduction was also performed using a cubic spline interpolation to calculate an $R^{2}$ coefficient. ${ }^{13}$ For this comparison, an aggregate metric of deviceassociated infections (which included all inpatient central line-associated bloodstream infections, catheter-associated urinary tract infections, and all intensive care unit-attributed ventilator-associated pneumonias) was used, because these infections were felt a priori to be impacted by $\mathrm{HH}$ adherence. Benchmark rates for each infection type were used to calculate an expected number of device-associated infections by unit type. ${ }^{14} \mathrm{~A}$ monthly composite device-associated standardized infection ratio (SIR) was calculated by dividing the total number of observed device-associated infections by the total number of expected infections. Similar to previously published work, ${ }^{15}$ surgical site infection data were excluded from the analysis, because these infections were expected to be inherently less sensitive to $\mathrm{HH}$ adherence. Because of migration to a nucleic acid amplification test for Clostridium difficile midway through the study period, this outcome was not included in the analysis, given that introduction of highersensitivity tests has been associated with increased infection rates. ${ }^{16}$

\section{RESULTS}

From July 2009 through August 2012, a total of 109,988 observations were completed, spanning 36 inpatient and 112 outpatient locations. Table 1 illustrates observations, mean number of observations, and mean $\mathrm{HH}$ adherence stratified by location for each program phase. Total numbers of observations obtained and $\mathrm{HH}$ adherence increased with each program phase. Overall adherence above $85 \%$ has been sustained since January 2011. Figure 2 illustrates the segmented regression analysis, showing differences in $\mathrm{HH}$ adherence over 


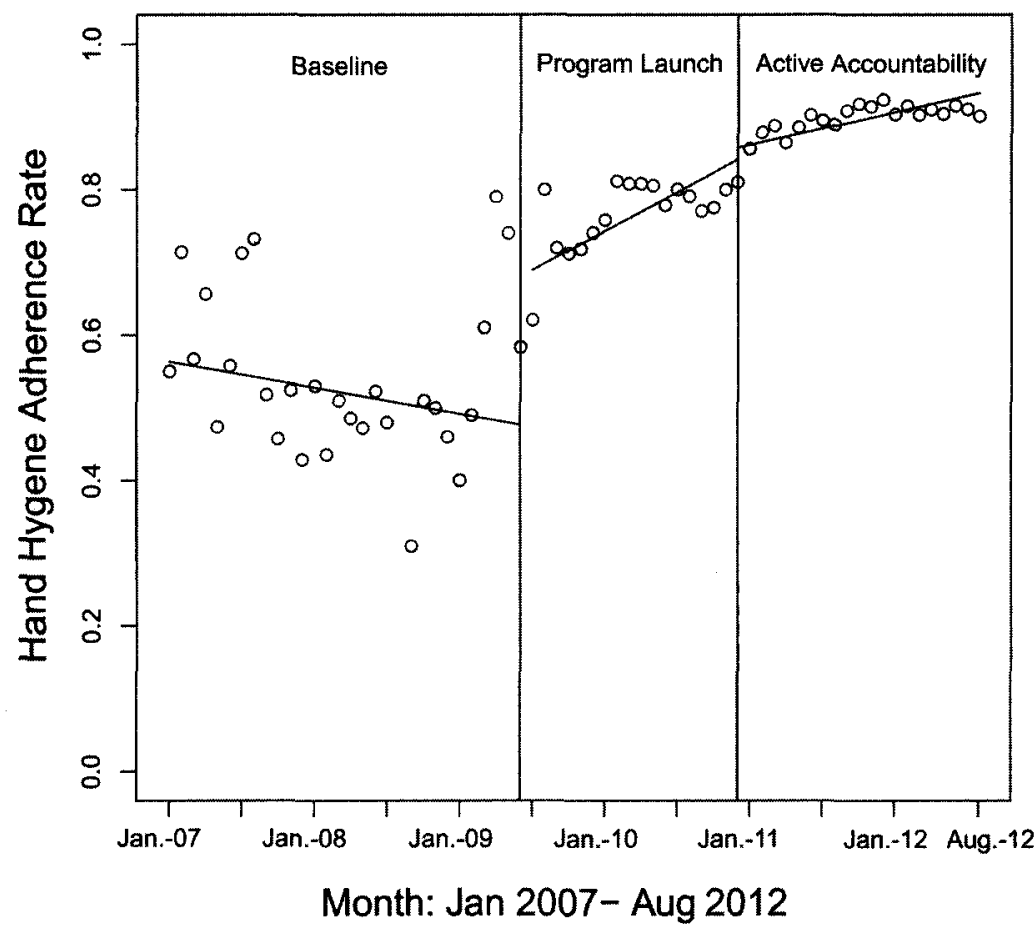

FIGURE 2. Segmented regression analysis evaluating monthly hand hygiene adherence rate (percentage adherent) over the 3 time periods included for study analysis: baseline, program launch, and active accountability. Each circle corresponds with the monthly institutional hand hygiene adherence rate. Each line corresponds with the calculated regression line for each time period.

time $(P<.0001)$, increases in adherence with each intervention phase $(P<.0001)$, and changes in the slope of the $\mathrm{HH}$ curves associated with each time period $(P<.032)$.

Table 2 illustrates the $\mathrm{HH}$ adherence by year, system performance, and percentage of available rebate dollars claimed. Leadership expected improvement and steadily "raised the bar." The target goal was achieved in FY 2010, permitting all units to reclaim $75 \%$ of rebate dollars. To date, a reach level of performance has not been achieved.

Figure 3 illustrates the relationship between monthly $\mathrm{HH}$ adherence and device-associated HAIs. $\mathrm{HH}$ adherence rates were inversely correlated with device-associated SIRs $\left(R^{2}=\right.$ $0.70)$. Both $\mathrm{HH}$ adherence and device-associated SIR showed significant improvement over time.

\section{I SCUSSION}

$\mathrm{HH}$ adherence improved with development and launch of an expanded direct observation program combined with goals and incentives promoting individual and group accountability. Improvements were supported by a process and method for sharing individual and unit data with interventions based on a $\mathrm{HH}$ accountability pyramid. ${ }^{7}$ High $\mathrm{HH}$ adherence has been sustained for 18 months. In addition, an impact on $\mathrm{HAI}$ rates is suggested, because $\mathrm{HH}$ adherence was inversely correlated with device-associated SIR. Specifically, in months in which institutional $\mathrm{HH}$ adherence rates were high, institution-wide device-associated SIRs were low. Interestingly, the shape of the correlation curve between $\mathrm{HH}$ adherence and infection rates suggests that only marginal improvement is achieved until a certain point is reached (75\% in our study). Because other HAI prevention initiatives were occurring, we cannot attribute causality to the decrease in device-associated infections during the study period. It is reasonable, however, to consider that improved $\mathrm{HH}$ rates had an impact. Most evidence-based bundles for reducing HAIs include $\mathrm{HH}$ as a component. It is also possible that $\mathrm{HH}$ marketing and accountability efforts reminded personnel of best practices in protecting patients from HAIs. Success of this multifaceted $\mathrm{HH}$ program is attributable to many factors, and several deserve review: obtaining leadership commitment, use of a tiered accountability model supporting a culture of safety, financial incentives, and the inclusion of outpatient and procedural areas.

Countless quality and safety initiatives are launched with good intent but wither on the vine because of a lack of effective leadership. ${ }^{5}$ Project leaders must continuously evaluate health system leadership support. Leadership commitment can be evaluated by asking a few questions. Is the project consistent with the system's quality and safety priorities? Does financial support exist, including support for key personnel? Are system leaders willing to publicly affirm commitment to the goal and its associated metrics? Are leaders willing to 
TABLE 2. Hand Hygiene Adherence and Self-Insurance Trust Rebate Performance

\begin{tabular}{|c|c|c|c|c|c|}
\hline \multirow{2}{*}{$\begin{array}{l}\text { Fiscal } \\
\text { year }^{\mathrm{a}}\end{array}$} & \multicolumn{3}{|c|}{ Performance goal, \% } & \multirow{2}{*}{$\begin{array}{c}\text { System } \\
\text { hand } \\
\text { hygiene } \\
\text { adherence, } \\
\%\end{array}$} & \multirow[t]{2}{*}{$\begin{array}{c}\text { Rebate, } \\
\% \text { of } \\
\text { available } \\
\text { potential } \\
\text { rebate } \\
\text { dollars } \\
\text { claimed }^{\text {b }}\end{array}$} \\
\hline & Threshold & Target & Reach & & \\
\hline 2010 & 65 & $75^{c}$ & 85 & 77 & 75 \\
\hline 2011 & $85^{c}$ & 90 & 95 & 85 & 50 \\
\hline 2012 & $88^{c}$ & 92 & 95 & 91 & 50 \\
\hline
\end{tabular}

"Fiscal year is defined as ending in June of the year listed (eg, fiscal year 2010 represents July 2009 through June 2010).

${ }^{b}$ Total potential rebate was $2.5 \%$ of yearly malpractice premiums (eg, if a department's premiums totaled $\$ 10,000$, the potential rebate for that department would be $\$ 250$ ).

c Correlates actual performance with the performance goal achieved.

address noncompliant individuals? Too often, system leadership support is signaled initially but then disappears once difficult decisions arise, undercutting the authority of the project team. Before initiating the VUMC HH program, leadership commitment was secured. When individuals resisted elements of the HH plan, VUMC leadership was prepared and responded in a measured and effective way.

Framing $\mathrm{HH}$ practices as a measure of professionalism supported accountability and feedback conversations associated with the $\mathrm{HH}$ program. Several physicians and nurses received accountability interventions on the basis of the $\mathrm{HH}$ accountability pyramid and the health system's professional conduct policy, and in each instance, leadership was fully supportive.

Shared accountability was present in the day-to-day function of the $\mathrm{HH}$ program at the location level. Each clinical area donated an observer to the observer pool who was responsible for observing practice in another area, creating shared reliance upon one another. Also, location-specific accountability interventions provided opportunities for hospital and clinic leadership to be invited in to partner with units to address underperformance. Additionally, the response of underperforming units' improvement plans provided leaders with insight into the safety culture in each poorly performing clinical area (eg, exhibiting a collegial mindset and desire to work as a team or, alternatively, dysfunction and a lack of leadership).

VUMC leadership also reasoned that financial incentives should align with achievement of important safety goals. Within the HH program, the potential rebate by departments and facilities amounted to $2.5 \%$ of yearly malpractice premiums, a small amount when distributed throughout the system. On the other hand, even small financial incentives impact medical practice behavior. ${ }^{17}$ Another aspect central to program success was the decision to have all departments and facilities share the same $\mathrm{HH}$ goals and therefore the same rebate percentage. Leaders were invested not only in the performance of their own areas but in the performance of other areas as well. This aspect of the program helped reduce a "silo" mentality. Sharing of unit-specific data also created collective accountability, because underperforming units were known by all whose rebate might be affected by their performance.

In addition to the traditional focus on inpatient wards, VUMC expanded the program focus to include outpatient and procedural areas using the same methodology throughout the system. Many HH programs either exclude outpatient clinics or use different metrics to assess their adherence. Although outpatient clinics outperformed inpatient units in overall $\mathrm{HH}$ adherence, a number of outpatient clinics and procedural areas required the tiered accountability approach. Procedural locations were also included. Although the workflow of the procedural locations provided challenges to leadership (eg, determining accessible locations for alcohol-based hand gel dispensers), these challenges also encouraged routine dialog about $\mathrm{HH}$ and its importance. In such circumstances, leadership went to great lengths to work through any barriers to $\mathrm{HH}$ adherence.

The VUMC program has limitations common to any observational $\mathrm{HH}$ surveillance program. First, direct observational programs are unable to observe most $\mathrm{HH}$ opportunities. ${ }^{18,19}$ Second, although each observer received the same training, an assessment of observer variability was not undertaken..$^{20}$ Third, this study is limited by its nonrandomized, quasi-experimental design, ${ }^{21}$ and we have no way to evaluate the impact of individual aspects of the program. In addition, observer bias likely influenced the results, and actual adherence may have been lower. By having the observers correct behavior, they clearly identified themselves, further increasing the risk of bias. ${ }^{22}$ Although such bias may influence an observational $\mathrm{HH}$ program, institutional leaders believed that the benefits of direct individual accountability outweighed the risk of artificially improved adherence. Although a direct observation $\mathrm{HH}$ program has well-described limitations, a hands-on direct feedback approach brings accountability benefits that are not possible with an entirely automated $\mathrm{HH}$ surveillance program. A final limitation is that many providers are not covered under self-insurance pools, a key component of our program's financial incentive; however, with changes in provider reimbursement, physicians are increasingly being employed by hospitals and, in most cases, being incorporated into their self-insurance programs. ${ }^{23}$ Thus, the applicability of the financial incentive used in the VUMC program may increase in the future.

\section{CONCLUSIONS}

We successfully implemented a multifaceted observational $\mathrm{HH}$ program based upon the principles of extensive project planning, leadership buy-in and goal setting, financial incentives linked to performance, and use of a system-wide shared 


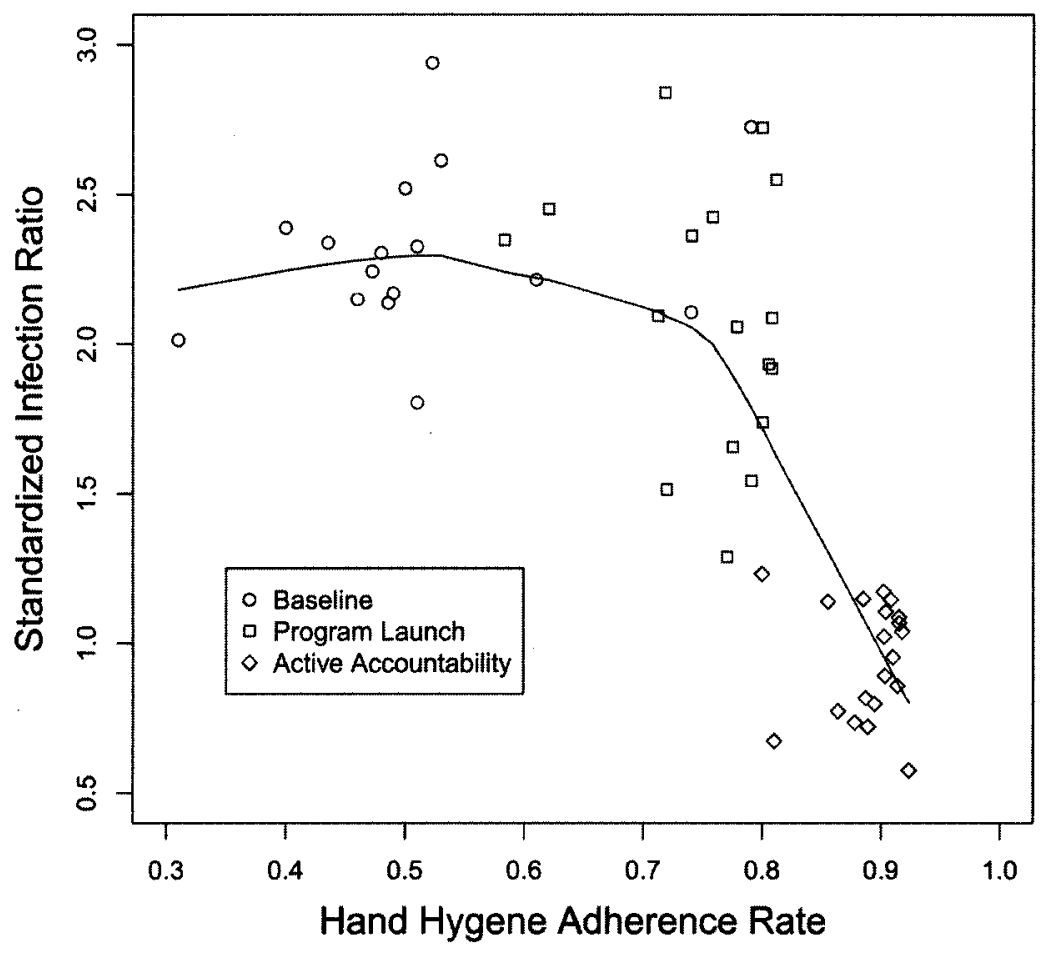

FIGURE 3. Correlation of monthly institutional hand hygiene rate (percentage adherent) with the corresponding institutional deviceassociated standardized infection ratio (SIR) is shown. Each point shown (circle, square, or diamond) corresponds to the hand hygiene adherence rate and device-associated SIR for the same month. Differing symbols denote each phase of the hand hygiene intervention. The fitted cubic spline interpolation line is shown.

accountability model. Improvements in HH have been sustained across the entire health system. Leadership engagement through a formal accountability structure coupled with institutional financial incentives have encouraged both nursing and physician leadership to pursue a culture of consistent, sustained $\mathrm{HH}$ adherence.

\section{ACKNOWLEDGMENTS}

We are indebted to all Vanderbilt University Medical Center (VUMC) faculty and staff for their participation in the hand hygiene program, especially for their enthusiasm and professionalism. We are indebted to all of the hand hygiene observers who make the VUMC hand hygiene program possible and to the hand hygiene executive committee members who guide the program. We would like to specifically acknowledge Ilene Moore, Brian Nelsen, Jim Pichert, Brian Rothman, Deede Wang, and the perioperative informatics team for their significant contributions to the VUMC hand hygiene program.

Potential conflicts of interest. All authors report no conflicts of interest relevant to this article. All authors submitted the ICMJE Form for Disclosure of Potential Conflicts of Interest, and the conflicts that the editors consider relevant to this article are disclosed here.

Address correspondence to Thomas R. Talbot, MD, MPH, Vanderbilt University School of Medicine, Department of Medicine, Division of Infectious Diseases, A2200 Medical Center North, 1161 21st Avenue South, Nashville, TN 37232 (tom.talbot@vanderbilt.edu).

\section{REFERENCES}

1. Larson E. A causal link between handwashing and risk of infection? examination of the evidence. Infect Control Hosp Epidemiol 1988;9(1):28-36.

2. Boyce JM, Pittet D; Society for Healthcare Epidemiology of America (SHEA)/Association for Professionals in Infection Control (APIC)/Infectious Diseases Society of America (IDSA). Guideline for hand hygiene in health-care settings: recommendations of the Healthcare Infection Control Practices Advisory Committee (HICPAC) and the HICPAC/SHEA/APIC/IDSA Hand Hygiene Task Force. MMWR Recomm Rep 2002;51(RR16):1-45.

3. McGuckin M, Waterman R, Govednik J. Hand hygiene compliance rates in the United States: a one-year multicenter collaboration using product/volume usage measurement and feedback. Am J Med Qual 2009;24(3):205-213.

4. Vanderbilt University Medical Center. Vanderbilt University Medical Center factbook, 2011-2012. http://www.mc.vanderbilt.edu/documents/main/files/2011 -2012VUMCfactbook.pdf. Accessed May 22, 2013.

5. Hickson GB, Moore IN, Pichert JW, Benegas M. Balancing systems and individual accountability in a safety culture. In: Berman S, ed. From Front Office to Front Line. 2nd ed. Oakbrook Terrace, IL: Joint Commission Resources, 2012:1-36.

6. Pittet D, Allegranzi B, Boyce J. The World Health Organization guidelines on hand hygiene in health care and their consensus 
recommendations. Infect Control Hosp Epidemiol 2009;30(7): 611-622.

7. Hickson GB, Pichert JW, Webb LE, Gabbe SG. A complementary approach to promoting professionalism: identifying, measuring, and addressing unprofessional behaviors. Acad Med 2007;82(11): 1040-1048.

8. Hickson GB, Federspiel CF, Pichert JW, Miller CS, Gauld-Jaeger J, Bost P. Patient complaints and malpractice risk. JAMA 2002; 287(22):2951-2957.

9. Hickson GB, Pichert JW. Identifying and addressing physicians at high risk for medical malpractice claims. In: Youngberg B, ed. Patient Safety Handbook. 2nd ed. Sudbury, MA: Jones and Bartlett Learning, 2012:347-368.

10. Horan TC, Andrus M, Dudeck MA. Centers for Disease Control and Prevention/National Healthcare Safety Network surveillance definition of health care-associated infection and criteria for specific types of infections in the acute care setting. Am J Infect Control 2008;36(5):309-332.

11. R: a language and environment for statistical computing. $R$ Foundation for Statistical Computing, 2012. http://www.R -project.org/. Accessed January 9, 2013.

12. Hmisc: Harrell miscellaneous. R package version 3.9, 2012. http://CRAN.R-project.org/package = Hmisc. Accessed January 9, 2013.

13. Wagner AK, Soumerai SB, Zhang F, Ross-Degnan D. Segmented regression analysis of interrupted time series studies in medication use research. I Clin Pharm Ther 2002;27(4):299-309.

14. Dudeck MA, Horan TC, Peterson KD, et al. National Healthcare Safety Network (NHSN) Report, data summary for 2010, deviceassociated module. Am J Infect Control 2011;39(10):798-816.

15. Kirkland KB, Homa KA, Lasky RA, Ptak JA, Taylor EA, Splaine ME. Impact of a hospital-wide hand hygiene initiative on health- care-associated infections: results of an interrupted time series. BMJ Qual Saf 2012;21(12):1019-1026.

16. Fong KS, Fatica $C$, Hall G, et al. Impact of PCR testing for Clostridium difficile on incident rates and potential on public reporting: is the playing field level? Infect Control Hosp Epidemiol 2011;32(9):932-933.

17. Hickson GB, Altemeier WA, Perrin JM. Physician reimbursement by salary or fee-for-service: effect on physician practice behavior in a randomized prospective study. Pediatrics 1987; 80(3):344-350.

18. Stewardson A, Pittet D. Quicker, easier, and cheaper? the promise of automated hand hygiene monitoring. Infect Control Hosp Epidemiol 2011;32(10):1029-1031.

19. Polgreen PM, Hlady CS, Severson MA, Segre AM, Herman T. Method for automated monitoring of hand hygiene adherence without radio-frequency identification. Infect Control Hosp Epidemiol 2010;31(12):1294-1297.

20. Aboumatar H, Ristaino $\mathrm{P}$, Davis RO, et al. Infection prevention promotion program based on the PRECEDE model: improving hand hygiene behaviors among healthcare personnel. Infect Control Hosp Epidemiol 2012;33(2):144-151.

21. Stewardson A, Pittet D. Anatomy of a successful multimodal hand hygiene campaign. BMJ Qual Saf 2012;21:973-975.

22. Rothlisberger FJ, Dickson WJ. Management and the worker: an account of a research program conducted by the Western Electric Company, Hawthorne Works, Chicago. Cambridge, MA: Harvard University Press, 1939.

23. American Society for Healthcare Risk Management. 2012 hospital and physician professional liability benchmark analysis. October 2012. http://www.aon.com/attachments/risk-services 12012-Aon-ASHRM-Benchmark-Report-Abridged-Version.pdf. Accessed May 22, 2013. 